\title{
Conformation of the Dipeptide Cyclo(L-Pro-L-Pro) Monitored by the Nuclear Magnetic Resonance and Raman Optical Activity Spectra. Experimental and ab Initio Computational Study
}

\author{
Petr Bouř, ${ }^{*}, \dagger$ Vladimír Sychrovský, ${ }^{\ddagger}$ Petr Maloň, ${ }^{\dagger}$ Jana Hanzlíková, ${ }^{\S}$ Vladimír Baumruk, ${ }^{\S}$ \\ Jan Pospíšek, and Miloš Buděšínský ${ }^{\dagger}$ \\ Institute of Organic Chemistry and Biochemistry, Academy of Sciences of the Czech Republic, \\ Flemingovo nám. 2, 166 10, Prague 6, Czech Republic, J. Heyrovský Institute of Physical Chemistry, \\ Center for Complex Molecular Systems and Biomolecules, Academy of Sciences of the Czech Republic, \\ Dolejškova 3, 182 23, Prague 8, Czech Republic, and Institute of Physics, Charles University, \\ Ke Karlovu 5, 121 16, Prague 2, Czech Republic
}

Received: July 12, 2001; In Final Form: May 17, 2002

\begin{abstract}
Nuclear magnetic resonance (NMR) and Raman optical activity (ROA) spectra of the cyclic dipeptide were measured and analyzed with respect to their ability to sense molecular structure and conformation. Data obtained by both techniques were simulated using ab initio quantum mechanical computations. Calculated chemical shifts, hydrogen-hydrogen spin-spin coupling constants and ROA intensities agreed well with the experimental values. The spin-spin NMR coupling constants were found to be most suitable for estimating of the conformational ratio. The ROA intensities provided additional information about the absolute configuration. The relation of the NMR chemical shifts to molecular structure was obscured by the solvent effect. The experimental results and calculated relative conformer energies suggest that equilibrium of three conformations takes place in the solution at the room temperature with a prevalence $(\sim 80 \%)$ of the conformation present in the crystalline state.
\end{abstract}

\section{Introduction}

Conformational equilibria of molecules in solutions are challenging both experimentally and theoretically. This study of the cyclo(L-Pro-L-Pro) dipeptide documents some interesting aspects of the problem encountered in the NMR and Raman optical activity (ROA) spectroscopies: (i) Both the NMR and ROA spectra reflect a second-order magnetic response of the electronic cloud in the molecule, but provide different and in many ways complementary information about the molecular structure. (ii) The size of the molecule and the tricyclic system severely limiting its flexibility are in favor of advanced computations. Similar compounds were found in nature, and supposedly, the results and methodology can be generalized to other peptides. (iii) Finally, we wanted to test the accuracy of the latest computational techniques developed for simulations of the NMR spin-spin coupling and ROA intensities.

Interpretations of NMR spectra are traditionally based on empirical relations between the chemical shifts, coupling constant patterns and structural fragments present in the molecule. For example, Karplus-like equations ${ }^{1}$ are extensively used for sugars or peptides, relating molecular conformation to vicinal spin-spin coupling constants. Obtained knowledge about the torsion angles of coupled nuclei may be supported by the measurement of the nuclear Overhauser effect (NOE). However, detailed relation between NMR spectrum in a given solvent and the structure and dynamics of the molecule is often unknown.

* To whom correspondence should be addressed. E-mail: bour@ uochb.cas.cz. Phone: (420-2)-20183-348. Fax: (420-2)-2431-0503.

$\dagger$ Institute of Organic Chemistry and Biochemistry.

$\doteqdot$ J. Heyrovský Institute of Physical Chemistry.

$\S$ Institute of Physics.
The conformation is determined to a large extent by relatively weak intramolecular nonbonded interactions as well as by solvent effects, which are traditionally difficult to model by empirical models. Thus more universal ab initio computational techniques are desirable for thorough interpretation of the experimental data.

Nuclear magnetic shielding and corresponding chemical shift can be calculated relatively easily by current quantum-chemical $\mathrm{ab}$ initio techniques. Typically, coupled-perturbed perturbation theories are used and an origin dependence of the results is avoided via the gauge-independent atomic orbitals (GIAOs). ${ }^{2}$ However, the dependence of the shifts on molecular conformation is often obscured by solvent effects or cannot be modeled with sufficient accuracy.

The NMR spin-spin coupling constants are more sensitive to subtle conformational changes than chemical shifts and supposedly less influenced by the solvent. Reliable calculation of the coupling is computationally more demanding and comprises a sum of four different contributions: ${ }^{3-5}$ the Fermi contact (FC), diamagnetic spin-orbit (DSO), paramagnetic spin-orbit (PSO), and spin-dipolar (SD) term. Moreover, electron correlation has to be included for their accurate simulation. The uncorrelated coupled Hartree-Fock (CHF) procedure, in many cases satisfactory for the shifts, consistently overestimated the constants by about 100-200\%. ${ }^{4}$ For smaller systems advanced post-HF perturbation procedures provided accurate constants and even reflected the solvent influence and molecular conformations. ${ }^{6}$ For larger molecules ( $>\sim 5$ atoms) application of these techniques were limited due to their demands on computing power, partially because of the lengthy numerical differentiation. Alternative procedures have been 
proposed, such a combination of ab initio computations and empirical Karlpus-like equations, ${ }^{7}$ or the analytical sum-overstates (SOS) scheme. ${ }^{4}$ Although the SOS method reproduced reasonably well signs and relative magnitudes of the coupling for many cases, it failed for accurate absolute values. Meanwhile, the analytical coupled-perturbed (CP) approach has been implemented at the DFT level, ${ }^{8,9}$ which enabled accurate computations of the coupling with more modest computer resources. Currently, we consider the analytical DFT/CP computation to be the best choice for larger systems and use it also for the cyclo(L-Pro-L-Pro). In our implementation, all the important terms in the magnetic Hamiltonian are included, which previously led to excellent results for a trial set of smaller molecules. The dipeptide provides another convenient benchmark, since the method has not yet been applied to larger and flexible molecules.

Unlike for NMR, interpretations of ROA spectra are almost entirely dependent on ab initio computational techniques. ${ }^{10}$ Natural complementarity of the two techniques stems also from the sensitivity of ROA to the absolute configuration that is not reflected by NMR spectra measured in achiral solvents. A close relation between molecular structure and ROA intensities has been established empirically for a variety of systems including carbohydrates, peptides, and nucleic acids. ${ }^{11,12}$ However, only a limited agreement between simulations and experiment was observed for more complex systems. Ab initio simulations are restricted to smaller molecules because of the lengthy numerical differentiation required for the construction of the electromagnetic tensor derivatives. ${ }^{13}$ Lately we proposed a computationally simpler and reasonably accurate simulation of ROA intensities ${ }^{10}$ based on the SOS expansions and use it also for cyclo(L-ProL-Pro) in this study. As shown below, accuracy of the SOS method is sufficient for ROA, unlike for the NMR spin-spin constants where it provides only informative values and the $\mathrm{CP}$ procedure should be applied.

Interestingly, all the spectral properties mentioned above reflect the electronic response to the magnetic perturbation caused either by the magnetic moments of the nuclei or by an external magnetic field. Moreover, the main NMR and ROA spectroscopic parameters are dependent on the second-order molecular property tensors. ${ }^{14}$ The NMR shielding tensor $\boldsymbol{\sigma}$, nuclear spin-spin coupling $\mathbf{J}$, and nuclear derivatives of the optical activity tensor $\mathbf{G}^{\prime}$ can be all written as second derivatives of the energy

$$
\begin{gathered}
\text { (NMR shielding) } \quad \boldsymbol{\sigma}=\left(\frac{\partial^{2} E}{\partial \mathbf{B} \partial m}\right)_{\mathbf{B}=\mathbf{0}, m=0} \\
\text { (NMR coupling) } \quad J_{n m}=\left(\frac{\partial^{2} E}{\partial \mathbf{n} \partial \mathbf{m}}\right)_{n=0, m=0} \\
\text { (ROA) } \quad \mathbf{G}^{\prime}=-\omega\left(\frac{\partial^{2} E}{\partial \dot{\mathbf{B}} \partial \mathbf{E}}\right)_{\dot{\mathbf{B}}=\mathbf{0}, \mathbf{E}=\mathbf{0}}
\end{gathered}
$$

where $E$ is the energy of the molecule, $\mathbf{B}$ external magnetic field, $\mathbf{E}$ electric intensity, $\omega$ frequency of the light, and $\mathbf{n}, \mathbf{m}$ nuclear magnetic moments. Scalar parameters $J_{n m}$ and $\sigma$ observable for an isotropic medium are defined as a 1/3 of the traces of the tensors $\mathbf{J}$ and $\boldsymbol{\sigma}$, respectively. For the ROA a backscattering incident circular polarization arrangement was used here and the corresponding intensity expression can be found elsewhere. ${ }^{14,15}$ The analogy of the NMR and ROA theories results also in similar problems associated with their computer implementation, e.g., the need of field-dependent atomic orbitals in order to avoid origin dependence of the results.

\section{Calculations}

Molecular geometry was optimized by energy minimization at the BPW916/6-311G** and levels using the Gaussian ${ }^{17}$ set of programs. The COSMO mode ${ }^{18}$ as implemented in Gaussian was used for estimation of the influence of aqueous environment $(\epsilon=78)$ on conformation energies. The NMR spin-spin coupling constants were obtained by the SOS method (for the BPW91/6-311G** and MP2/6-31G** geometries) with the Becke-3LYP (B3LYP) ${ }^{19}$ functional and 6-311G** basis functions using the program $\mathrm{Roa}^{4}$ written in house, and at the CP/ B3LYP/6-31G** level using the COLOGNE 99 program package $^{20}$ with the modified atomic basis of Huzinaga ${ }^{21}$ (9s $5 \mathrm{p} 1 \mathrm{~d} /$ $5 \mathrm{~s}, 1 \mathrm{p})[6 \mathrm{~s}, 4 \mathrm{p}, 1 \mathrm{~d} / 3 \mathrm{~s}, 1 \mathrm{p}]$, usually referred to as IGLO-II. The ROA intensities were simulated using harmonic force field computed by Gaussian using the BPW91/6-311G** procedure. Finally, the ROA tensors derivatives ${ }^{14}$ were constructed from the Becke3LYP/SOS/6-31++ $\mathrm{G}^{* *}$ derivatives of the $\mathbf{G}^{\prime}$ and $\mathbf{A}$ (dipolequadrupole polarizability) tensors combined with the dipole polarizability $\alpha$ computed at the $\mathrm{HF} / \mathrm{CP} / 6-31++\mathrm{G}^{* *}$ level using a method described elsewhere. ${ }^{10}$ Due to the lack of reliable theoretical methods, solvent corrections were not attempted for the simulations of the NMR and ROA parameters.

\section{Experimental Section}

Synthesis. The dipeptide was prepared by a standard fragment condensation. ${ }^{22}$ Purity of the resultant compound was better then $98 \%$.

NMR Spectroscopy. NMR spectra of cyclo were measured on the Varian UNITY-500 spectrometer $\left({ }^{1} \mathrm{H}\right.$ at $500 \mathrm{MHz} ;{ }^{13} \mathrm{C}$ at $125.7 \mathrm{MHz},{ }^{17} \mathrm{O}$ at $67.8 \mathrm{MHz}$ and ${ }^{15} \mathrm{~N}$ at $50.7 \mathrm{MHz}$ frequency) in $\mathrm{CDCl}_{3}$. For estimation of the solvent effect on chemical shifts the ${ }^{1} \mathrm{H}$ and ${ }^{13} \mathrm{C}$ NMR spectra were measured also in $\mathrm{C}_{6} \mathrm{D}_{6}, \mathrm{CD}_{3^{-}}$ $\mathrm{OD}, \mathrm{CD}_{3} \mathrm{SOCD}_{3}$ and $\mathrm{D}_{2} \mathrm{O}$. All measurements were performed at the room temperature $(\sim 298 \mathrm{~K})$. Concentrations of about 5 mg of peptide in $0.5 \mathrm{~mL}$ solution was used for all ${ }^{1} \mathrm{H}$ and ${ }^{13} \mathrm{C}$ NMR spectra and $50 \mathrm{mg} / 0.5 \mathrm{~mL}$ for ${ }^{17} \mathrm{O}$ and ${ }^{15} \mathrm{~N}$ NMR. ${ }^{1} \mathrm{H}$ and ${ }^{13} \mathrm{C}$ chemical shifts are referenced to internal tetramethylsilane (TMS) in case of organic solvents and/or to sodium salt of 3-(trimethylsilyl)-1-propane sulfonic acid (DSS) in case of $\mathrm{D}_{2} \mathrm{O}$. For ${ }^{15} \mathrm{~N}$ and ${ }^{17} \mathrm{O}$ chemical shifts a secondary referencing to nitromethane and dioxane, respectively, was applied. Proton chemical shifts and interproton coupling constants extracted from resolution enhanced NMR spectra were refined by simulation-iteration procedure (using program gNMR V4.0). The vicinal $\mathrm{J}(\mathrm{H}, \mathrm{H})$ of the proline ring were interpreted in terms of pseudorotation analysis. ${ }^{23,24}$ This approach combines the generalized Karplus equation, ${ }^{25}$ the concept of pseudorotation ${ }^{26}$ and a two-state model in a least-squares procedure. Calculations were performed with the program CONFIT. ${ }^{27}$

ROA Experiment. Cyclo(L-Pro-L-Pro) was dissolved in doubly distilled deionized water to the final concentration of about $1 \mathrm{~mol} / \mathrm{L}$. Back-scattered ICP ROA and Raman spectra were collected at the room temperature on the instrument built at the Charles University described in detail elsewhere. ${ }^{28,29}$ The spectra were excited with the $514.5 \mathrm{~nm}$ line of argon laser (Coherent Innova 305). The excitation power at the sample was about $500 \mathrm{~mW}$; the width of the spectrograph entrance slit was set to $200 \mu \mathrm{m}$ corresponding to a spectral width of about 10 $\mathrm{cm}^{-1}$, the total acquisition time was $4.5 \mathrm{~h}$. Experimental data are displayed in the form of circular intensity difference, $I^{\mathrm{R}}-$ $I^{\mathrm{L}}$, representing the ROA spectrum, and a corresponding circular intensity sum, $I^{\mathrm{R}}+I^{\mathrm{L}}$, representing the parent Raman spectrum (where $I^{\mathrm{R}}$ and $I^{\mathrm{L}}$ are the Raman scattered intensities with right and left circularly polarized incident light, respectively). The 


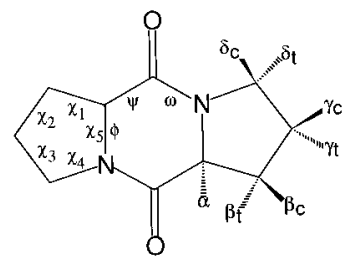

Figure 1. Atom and torsion angle definitions in cyclo(L-Pro-L-Pro).
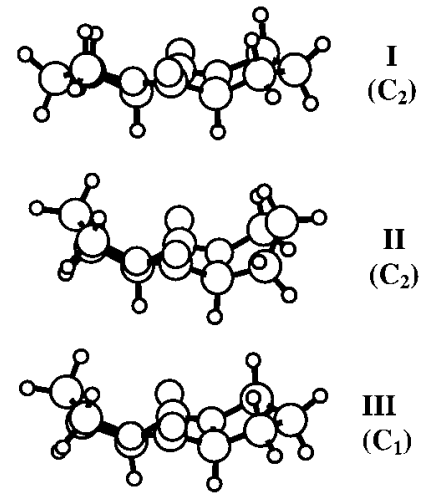

Figure 2. Optimized (BPW91/6-311G**) geometries of the three conformers (molecular symmetry is indicated in parentheses).

opposite enantiomer, cyclo(D-Pro-D-Pro), was also studied at the same experimental conditions and its ROA spectrum exhibited almost perfect mirror symmetry. ${ }^{29}$

\section{Results and Discussion}

Molecular Conformers. Three equilibrium conformers referred to as I, II, and III (shown in Figure 2) were found for cyclo(L-Pro-L-Pro). Their characteristic torsion angles (see Figure 1) calculated at the BPW91/6-311G** and MP2/6-31G** levels are compared with experimental values in Table 1. Apparently, conformation I corresponds to that found by the
$\mathrm{X}$-ray in crystal $^{30}$ as well as to the $\mathrm{N}$ (North)-conformation obtained by the pseudorotation analysis of NMR data. ${ }^{23,27}$ Conformation II, corresponding to the S (South) conformation obtained by NMR, was not observed in the crystalline state. Both conformations I and II possess a $C_{2}$ symmetry axis, while conformation III loses the symmetry and can be viewed as an intermediate state between the first two forms (see Figure 2). In contrast to the five-membered rings the inner six-membered ring is relatively rigid and not influenced by the conformation of the side chains (compare angles $\phi, \iota$, and $\omega$ in Table 1). For conformer I, most of the angles (except $\chi_{1}$ ) obtained at the BPW91/6-31G** level are closer to the X-ray data than those calculated by the MP2/6-31G** method, but this difference is minor with respect to the experimental error. Also, the effect of crystal forces could not be involved in the computations.

Calculated Relative Energies. Calculated relative energies of the three conformers are listed in Table 2. Clearly, the differences between the conformers are small and inconsistent even if calculated at relatively high levels of approximations. For example, the correlated methods of MP2 and BPW91 provide the same ordering of electronic energies, but different from that obtained by the HF procedure. For BPW91, virtually same energies are obtained with the two basis sets $\left(6-31 \mathrm{G}^{* *}\right.$ and $\left.6-311 \mathrm{G}^{* *}\right)$, but the ordering is changed if the COSMO solvent model $\left(\epsilon_{\mathrm{r}}=78\right)$ is used. The B3LYP functional provides virtually same electronic energies for all the three conformers. The ordering, however, is changed again when the vibrational zero-point energy correction is considered or the Gibbs free energies are compared. Thus the calculation of relative energies alone does not provide reliable information on the conformer populations in the sample. Since, however, the differences in relative energies are comparable with the Boltzmann quantum (at the room-temperature $k T \sim 0.6 \mathrm{kcal} / \mathrm{mol}$ ), a semifree movement of the proline rings can be deduced.

TABLE 1: Calculated and Experimental Ring Torsion Angles in Cyclo(L-Pro-L-Pro)

\begin{tabular}{|c|c|c|c|c|c|c|c|c|c|c|c|}
\hline & \multicolumn{4}{|c|}{ BPW91/6-311G** } & \multicolumn{4}{|c|}{$\mathrm{MP} 2 / 6-31 \mathrm{G}^{* *}$} & \multirow[b]{2}{*}{ X-ray ${ }^{b}$} & \multicolumn{2}{|c|}{$\mathrm{NMR}^{c}$} \\
\hline & conformer I & conformer II & confo & er III ${ }^{a}$ & conformer $\mathbf{I}$ & conformer II & confor & $\operatorname{er} \mathbf{I I I}^{a}$ & & $\mathrm{~N}$-form & S-form \\
\hline$\varphi$ & -36.6 & -39.3 & -38.0 & -36.9 & -35.9 & -41.0 & -39.2 & -39.2 & -38 & & \\
\hline$\psi$ & 38.1 & 44.5 & 42.3 & 41.3 & 39.4 & 48.7 & 44.6 & 44.6 & 37 & & \\
\hline$\omega$ & -2.3 & -6.0 & -4.7 & -5.7 & -4.6 & -8.3 & -6.3 & -6.4 & -1 & & \\
\hline$\chi_{1}$ & -31.1 & 19.4 & -29.7 & 18.1 & -32.5 & 25.7 & -30.0 & 23.7 & -34 & -34 & 34 \\
\hline$\chi_{2}$ & 37.1 & -33.8 & 36.9 & -33.0 & 39.4 & -38.7 & 39.1 & -37.9 & 36 & 39 & -43 \\
\hline$\chi_{3}$ & -28.1 & 34.9 & -29.2 & 35.0 & -30.5 & 36.1 & -32.2 & 36.9 & -24 & -29 & 36 \\
\hline$\chi_{4}$ & 8.7 & -24.0 & 10.9 & -25.0 & 10.4 & -21.0 & 13.8 & -23.2 & 1 & 9 & -15 \\
\hline$\chi_{5}$ & 14.3 & 3.0 & 11.9 & 4.4 & 13.8 & -3.0 & 10.3 & -0.3 & 21 & 15 & -12 \\
\hline
\end{tabular}

${ }^{a}$ For this conformer the two proline residues are not identical. ${ }^{b}$ Reference $30 .{ }^{c}$ The analysis deals with the five-membered rings only.

TABLE 2: Calculated Relative Energies (kcal/mol) of the Conformers

\begin{tabular}{ccccccc}
\hline \multicolumn{5}{c}{ (a) Electronic Energies $(E)$} \\
\hline conformer & HF 6-31G** & MP2 6-31G** & BPW91 6-31G** & BPW91 6-311G** & BPW91/COSMO $^{a}$ 6-311G BP $^{* *}$ & B3LYP 6-31G** $^{* *}$ \\
\hline I & 0 & 0 & 0 & 0 & 0 & 0 \\
II & 0.8 & -0.9 & -0.5 & -0.5 & 0.7 & -0.1 \\
III & 1.4 & -0.3 & -0.2 & -0.3 & 0.7 & 0.0
\end{tabular}

(b) With Zero-Point Energies (ZPE) and Free Energies at $298 \mathrm{~K}(\Delta G)$

\begin{tabular}{|c|c|c|c|c|c|c|}
\hline \multirow[b]{2}{*}{ conformer } & \multicolumn{2}{|c|}{$\mathrm{HF} / 6-31 \mathrm{G}^{* *}$} & \multicolumn{2}{|c|}{ BPW91/6-31G** } & \multicolumn{2}{|c|}{ B3LYP/6-31G** } \\
\hline & $E+\mathrm{ZPE}$ & $\Delta G$ & $E+\mathrm{ZPE}$ & $\Delta G$ & $E+\mathrm{ZPE}$ & $\Delta G$ \\
\hline I & 0 & 0 & 0 & 0 & 0 & 0 \\
\hline II & 0.9 & 0.6 & -0.1 & 0.5 & 0.3 & -0.8 \\
\hline III & 1.7 & 1.9 & -0.1 & -0.2 & 0.2 & 0.0 \\
\hline
\end{tabular}

${ }^{a}$ Water as a solvent was used, with default Gaussian parameters. 
TABLE 3: Experimental and Calculated Chemical Shifts (ppm)

\begin{tabular}{|c|c|c|c|c|c|c|c|c|c|}
\hline \multirow[b]{2}{*}{ nuclei } & & \multicolumn{5}{|c|}{ experimental } & \multicolumn{3}{|c|}{ calculated for conformer: } \\
\hline & & $\mathrm{CDCl}_{3}$ & $\mathrm{C}_{6} \mathrm{D}_{6}$ & DMSO & $\mathrm{CD}_{3} \mathrm{OD}$ & $\mathrm{D}_{2} \mathrm{O}$ & $\mathbf{I}$ & II & III $^{a}$ \\
\hline \multirow[t]{5}{*}{${ }^{13} \mathrm{C}^{b}$} & $\mathrm{CO}$ & 166.40 & 165.91 & 165.90 & 168.63 & 171.02 & 164.2 & 163.0 & 164.2 \\
\hline & $\mathrm{C} \alpha$ & 60.56 & 60.32 & 59.66 & 61.76 & 63.41 & 67.6 & 66.4 & 66.9 \\
\hline & $\mathrm{C} \beta$ & 27.70 & 27.88 & 27.10 & 28.74 & 29.97 & 33.3 & 30.6 & 31.9 \\
\hline & $\mathrm{C} \gamma$ & 23.36 & 23.22 & 22.78 & 24.19 & 25.37 & 28.8 & 28.7 & 28.7 \\
\hline & $\mathrm{C} \delta$ & 45.22 & 45.11 & 44.50 & 46.21 & 47.94 & 50.2 & 51.1 & 50.6 \\
\hline \multirow[t]{7}{*}{${ }^{1} \mathrm{H}^{b}$} & $\mathrm{H} \alpha$ & 4.10 & 3.54 & 4.28 & 4.35 & 4.44 & 4.23 & 4.04 & 4.12 \\
\hline & $\mathrm{H} \beta_{\mathrm{c}}$ & 2.31 & 2.13 & 2.12 & 2.29 & 2.32 & 2.07 & 2.99 & 2.57 \\
\hline & $\mathrm{H} \beta_{\mathrm{t}}$ & 2.19 & 1.94 & 1.93 & $\sim 2.00$ & 2.09 & 2.10 & 1.85 & 1.97 \\
\hline & $\mathrm{H} \gamma_{\mathrm{c}}$ & 2.02 & 1.34 & $\sim 1.86$ & $\sim 2.00$ & $\sim 2.00$ & 1.95 & 1.55 & 1.75 \\
\hline & $\mathrm{H} \gamma_{\mathrm{t}}$ & 1.93 & 1.21 & 1.82 & $\sim 2.00$ & $\sim 2.00$ & 1.87 & 1.63 & 1.75 \\
\hline & $\mathrm{H} \delta_{\mathrm{c}}$ & 3.53 & 3.36 & $\sim 3.35$ & $\sim 3.52$ & 3.56 & 3.36 & 4.14 & 3.75 \\
\hline & $\mathrm{H} \delta_{\mathrm{t}}$ & 3.53 & 3.21 & $\sim 3.35$ & $\sim 3.48$ & 3.46 & 3.65 & 3.05 & 3.36 \\
\hline${ }^{15} \mathrm{~N}^{c}$ & $\mathrm{~N}$ & -249.29 & & & & & -226.1 & -223.6 & -225.0 \\
\hline${ }^{17} \mathrm{O}^{d}$ & $\mathrm{O}$ & 302.87 & & & & & 346.8 & 337.7 & 342.2 \\
\hline
\end{tabular}

${ }^{a}$ The average value for the two proline residues. ${ }^{b}$ Referenced to TMS. ${ }^{c}$ Referenced to nitromethane. ${ }^{d}$ Referenced to dioxane. The BPW91/6$311 \mathrm{G}^{* *}$ level was used for the computation, at which the absolute reference values were calculated as 183.34 (C, TMS), 31.67 (H, TMS), -120.68 ( $\mathrm{N}$, nitromethane), and $269.83(\mathrm{O}$, dioxane) $\mathrm{ppm}$.

TABLE 4: NMR Spin-Spin Coupling Constants ${ }^{a}$ in Cyclo(Pro-Pro), in Hz

\begin{tabular}{|c|c|c|c|c|c|c|c|c|c|c|c|c|}
\hline \multirow[b]{3}{*}{$\mathrm{H}, \mathrm{H}$} & \multirow[b]{3}{*}{$n$} & \multicolumn{11}{|c|}{${ }^{n} J(\mathrm{H}, \mathrm{H})$} \\
\hline & & \multicolumn{2}{|c|}{ SOS/6-311G**b } & \multicolumn{2}{|c|}{$\mathrm{CP} / 6-31 \mathrm{G}^{c}$} & \multicolumn{4}{|c|}{ CP/IGLO-II ${ }^{c}$} & \multicolumn{2}{|c|}{ CP/IGLO-II ${ }^{b}$} & \multirow{2}{*}{$\begin{array}{c}\text { exptl } \\
\text { (in } \mathrm{C}_{6} \mathrm{D}_{6} \text { ) }\end{array}$} \\
\hline & & I & II & I & II & I & II & III & III' $^{\prime}$ & I & II & \\
\hline$\beta_{\mathrm{c}} \beta_{\mathrm{t}}$ & 2 & -0.15 & 0.16 & -17.78 & -16.81 & -13.27 & -12.21 & -13.42 & -12.39 & -13.44 & -12.77 & -12.90 \\
\hline$\gamma_{\mathrm{c}} \gamma_{\mathrm{t}}$ & 2 & 0.00 & 0.15 & -17.06 & -16.80 & -12.52 & -12.21 & -12.44 & -12.17 & -12.79 & -12.38 & -12.65 \\
\hline$\delta_{\mathrm{c}} \delta_{\mathrm{t}}$ & 2 & 0.54 & 0.47 & -16.28 & -16.15 & -11.57 & -11.47 & -11.39 & -11.49 & -12.03 & -11.83 & -11.58 \\
\hline$\alpha \beta_{\mathrm{c}}$ & 3 & 4.52 & 0.48 & 9.32 & 0.48 & 9.76 & 0.54 & 9.51 & 0.67 & 9.85 & 1.09 & 8.82 \\
\hline$\alpha \beta_{\mathrm{t}}$ & 3 & 2.38 & 1.01 & 7.40 & 9.90 & 6.33 & 8.62 & 6.70 & 9.05 & 6.39 & 9.67 & 7.37 \\
\hline$\beta_{\mathrm{c}} \gamma_{\mathrm{c}}$ & 3 & 2.48 & 2.72 & 7.03 & 7.44 & 6.07 & 6.27 & 6.18 & 6.44 & 6.66 & 7.43 & 7.36 \\
\hline$\beta_{\mathrm{c}} \gamma_{\mathrm{t}}$ & 3 & 5.69 & 0.18 & 12.70 & 0.57 & 12.71 & 0.61 & 12.81 & 0.56 & 12.52 & 0.42 & 10.29 \\
\hline$\beta_{\mathrm{t}} \gamma_{\mathrm{c}}$ & 3 & 0.22 & 5.29 & 0.57 & 11.80 & 0.63 & 12.00 & 0.61 & 12.02 & 0.47 & 11.70 & 3.80 \\
\hline$\beta_{\mathrm{t}} \gamma_{\mathrm{t}}$ & 3 & 2.34 & 2.61 & 6.69 & 6.86 & 5.78 & 6.02 & 5.88 & 6.18 & 6.33 & 6.92 & 6.84 \\
\hline$\gamma_{\mathrm{c}} \delta_{\mathrm{c}}$ & 3 & 2.61 & 2.65 & 7.34 & 8.53 & 6.38 & 7.17 & 6.07 & 6.99 & 6.96 & 7.32 & 7.36 \\
\hline$\gamma_{\mathrm{c}} \delta_{\mathrm{t}}$ & 3 & 0.28 & 5.09 & 0.15 & 10.86 & 0.37 & 11.04 & 0.34 & 10.98 & 0.53 & 10.98 & 3.90 \\
\hline$\gamma_{t} \delta_{c}$ & 3 & 4.87 & 0.19 & 10.50 & 0.15 & 10.81 & 0.31 & 10.92 & 0.31 & 10.48 & 0.32 & 8.43 \\
\hline$\gamma_{t} \delta_{t}$ & 3 & 3.25 & 2.08 & 9.59 & 6.04 & 8.33 & 5.16 & 8.04 & 5.02 & 8.86 & 5.47 & 8.40 \\
\hline$\alpha \delta_{\mathrm{c}}$ & 4 & 0.48 & 0.43 & -1.81 & -1.75 & -0.76 & -0.74 & -0.78 & -0.74 & -0.77 & -0.76 & -0.72 \\
\hline$\alpha \delta_{t}$ & 4 & 0.10 & -0.01 & -1.06 & -0.81 & -0.63 & -0.57 & -0.68 & -0.52 & -0.60 & -0.53 & -0.59 \\
\hline$\alpha \gamma_{\mathrm{c}}$ & 4 & 0.37 & 0.38 & -1.51 & -1.55 & -0.59 & -0.61 & -0.64 & -0.61 & -0.58 & -0.61 & -0.50 \\
\hline$\beta_{\mathrm{c}} \delta_{\mathrm{c}}$ & 4 & -0.24 & 1.10 & 0.43 & 0.11 & -0.18 & 0.99 & -0.17 & 1.08 & -0.20 & 0.91 & 0.60 \\
\hline$\beta_{\mathrm{t}} \delta_{\mathrm{c}}$ & 4 & 0.37 & 0.38 & -1.64 & -1.54 & -0.68 & -0.55 & -0.65 & -0.55 & -0.70 & -0.64 & -0.68 \\
\hline$\beta_{\mathrm{t}} \delta_{\mathrm{t}}$ & 4 & 0.94 & -0.24 & -0.21 & 0.48 & 0.63 & -0.16 & 0.68 & -0.16 & 0.45 & -0.19 & 0.62 \\
\hline$\eta(\%)^{d}$ & & $71^{e}$ & $29^{e}$ & 77 & 23 & 77 & 23 & 77 & 23 & 78 & 22 & \\
\hline
\end{tabular}

${ }^{a}$ All constants were calculated using the B3LYP functional. ${ }^{b}$ BPW91/6-311G** optimized geometry. ${ }^{c}$ MP2/6-31G** geometry. ${ }^{d}$ Calculated molar ratios obtained by a root-mean-square fitting to the experimental values; two-state model is adopted; for conformer III the five membered rings are considered separately. ${ }^{e}$ Arbitrarily normalized.

Isotropic Nuclear Magnetic Shielding. Calculated and experimental chemical shifts are listed in Table 3. Typically, computed values agree with experiment within an error of about $10 \%$. The chemical shifts of heavy atoms seem to be relatively less sensitive to the change of conformation than for hydrogen atoms. For example, computed shifts for the hydrogen $\mathrm{H} \beta_{\mathrm{c}}$ differ by $0.92 \mathrm{ppm}$ (about $50 \%$ ) for conformations I and II, while for carbons the biggest difference of $2.7 \mathrm{ppm}\left(\mathrm{C}_{\beta}\right)$ corresponds to only about $10 \%$ of the absolute value and is comparable with expected computational error. Unfortunately, as follows from comparison with the experimental values in Table 3 , the conformational dependence predicted for the hydrogen atoms has same magnitude as the changes induced by the solvent. We suppose that the chemical shifts obtained with chloroform are most suitable for comparison with the data calculated for vacuum, because of the weak solvent-solute interactions. Although benzene is less polar than $\mathrm{CDCl}_{3}$, it typically induces large changes in the shifts. Using the chloroform data, the root- mean-square deviations between the calculated and experimental proton chemical shifts $(0.14$ and $0.46 \mathrm{ppm}$ for conformations I and II, respectively) indicate that conformer $\mathbf{I}$ is preferred in the sample.

Nuclear Spin-Spin Coupling. The coupling constants (namely, the vicinal couplings) are known to be very sensitive to conformational changes. Indeed, as follows from Table 4, significant differences can be observed in the coupling constants calculated for the two conformations of the proline side chain. An occurrence of conformer III of cyclo(Pro-Pro) cannot be evidenced by experimental NMR data, since one of its proline ring adopts geometry present in conformer $\mathbf{I}$ and the second proline ring corresponds to conformer II. In the time-averaged experimental spectrum the presence of conformer III apparently increases effective ratios of the conformers I and II by the same amount. Thus we adopt a two state model, which is also supported by the small energy differences (see Table 2) among the conformers. Note, that the relative energies not only suggest 
independent movement of the two proline rings, but also their coupling constants in conformer III (columns titled III and III in Table 4) virtually copy those calculated for the "pure" conformers I and II. For example, the $\alpha \beta_{\text {c }}$ coupling constant was obtained as 9.76 with the CP/IGLO-II ${ }^{\mathrm{b}}$ model for conformer I. This number is quite close to the value of 9.51 obtained by the same method for the proline ring adopting similar (crystalline-like) conformation in the conformer III. The other conformational minimum of the five member ring leads to a quite different value of this constant, 0.54 and 0.67 , predicted for the conformers II and III, respectively.

The SOS values for couplings between the hydrogen atoms separated only by two covalent bonds $(n=2)$ are clearly quite unrealistic, in accord with our previous experience, ${ }^{4,8}$ This shortrange coupling is much better reproduced by the $\mathrm{CP}$ technique (columns 5-12 in Table 4), especially when the larger "NMRoptimized" IGLO-II basis set is used. Control computations (not shown here) confirmed the general knowledge that the couplings are not significantly influenced by the solvent, e.g., by formation of hydrogen bonds, and thus can be really considered dependent predominantly on molecular structure and conformation.

Calculated values of the coupling constants over more than two bonds $(n>2)$ are generally less sensitive to the adopted approximation. In accordance with previous observations ${ }^{4,8}$ these constants obtained with the approximate SOS method follow the $\mathrm{CP}$ values, but are underestimated by about $50 \%$. The CP constants for $n=3$ obtained with the smaller basis (columns 5-6) are very close to those calculated with the bigger basis set (columns $7-8$ in Table 4); more pronounced basis set dependence appears for the longest range interactions $(n=4)$. Rather minor differences can be observed for the CP/IGLO-II ${ }^{\mathrm{a}}$ and CP/IGLO-II ${ }^{\mathrm{b}}$ computations, where the BPW91/6-311G** and $\mathrm{MP} 2 / 6-31 \mathrm{G}^{* *}$ equilibrium geometries were used, respectively.

Since the experimental spin-spin coupling constants are not reasonably matched by the prediction for the pure conformers, we suppose that a conformer equilibrium is present in the sample. Then the experimentally observed coupling constants correspond to a population-weighted sum of the $J$-values of individual conformers. Adopting the two-state model (for the proline rings rather than molecular conformers), sum of the square deviations between experimental and calculated couplings can be minimized,

$$
\delta=\Sigma_{i}\left[J_{i}(\exp )-\left(\eta^{\mathbf{I}} J_{i}(\mathbf{I})+\eta^{\mathbf{I I}} J_{i}(\mathbf{I I})\right)\right]^{2} \rightarrow \min
$$

which provides the mole fractions $\eta^{\mathrm{i}}$ of the conformers. Because of the large systematic error of the SOS method, the normalization $\left(\eta^{\mathbf{I}}+\eta^{\mathbf{I I}}=1\right)$ was introduced arbitrarily after the fitting for this case. As can be judged from Table 4 and Figure 3 , such a fit (with $\eta^{\mathbf{I}} \sim 0.78$ ) provides a perfect agreement with the experimental data. Obtained molar ratios (for the $\mathrm{CP}$ computations) are virtually independent of the basis set and on minor differences between the BPW91 and MP2 geometries. This result is also in agreement with the pseudorotation analysis based on the observed vicinal couplings since the generalized Karplus equation and a similar two-state model for proline conformation predict about $80 \%$ of conformation $\mathbf{I}$ in the solution of cyclo(Pro-Pro). At the time-averaging situation the presence of conformation III will result in apparent increasing of conformations I and II by the same amount and the population of III itself cannot be therefore determined by the NMR data.

Raman Optical Activity. By this technique, in principle, all the conformations present in the sample should be resolved.
H-H Coupling Constants $(\mathrm{Hz})$ in C-(L-Pro-L-Pro)

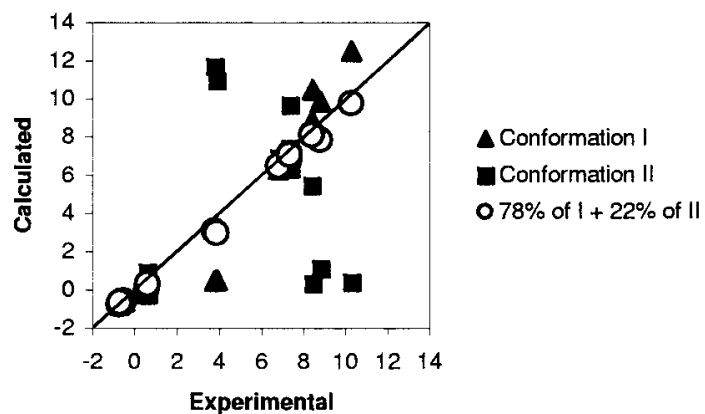

Figure 3. Calculated and experimental nuclear spin-spin coupling constants. Only the longer range coupling $(n>2$, see Table 4$)$ is included.

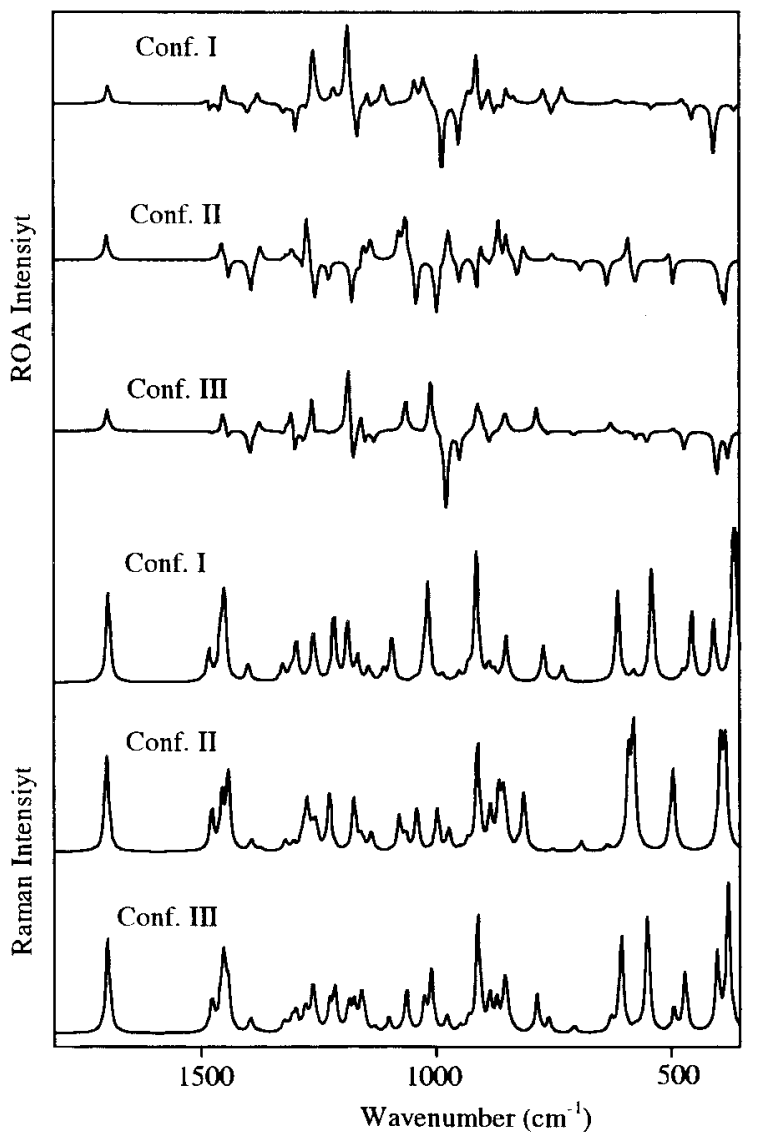

Figure 4. Simulated Raman (lower part) and ROA (upper part) spectra of the three conformations of cyclo(L-Pro-L-Pro).

Unlike NMR, due to the fast intrinsic response of ROA given by the light frequency, the conformations contribute separately to the spectrum. As follows from the simulation of the ROA and Raman spectra shown in Figure 4, the three forms of cyclo(Pro-Pro) provide unique signals almost in the entire range of recorded frequencies. However, a lower sensitivity of the technique as well as limited accuracy of the simulation does not allow full exploration of this potential. Only calculated spectrum of conformer I provided satisfying agreement with experimental spectrum, as shown in Figure 5. In fact, the agreement is very good and a standard analysis would lead to the conclusion that only conformer I was present in the sample. Under this assumption most of the visible spectral features can be assigned to fundamental transitions as done in Table 5. Obviously, the correspondence between the experimental and simulated ROA sign patterns reliably confirms the absolute 


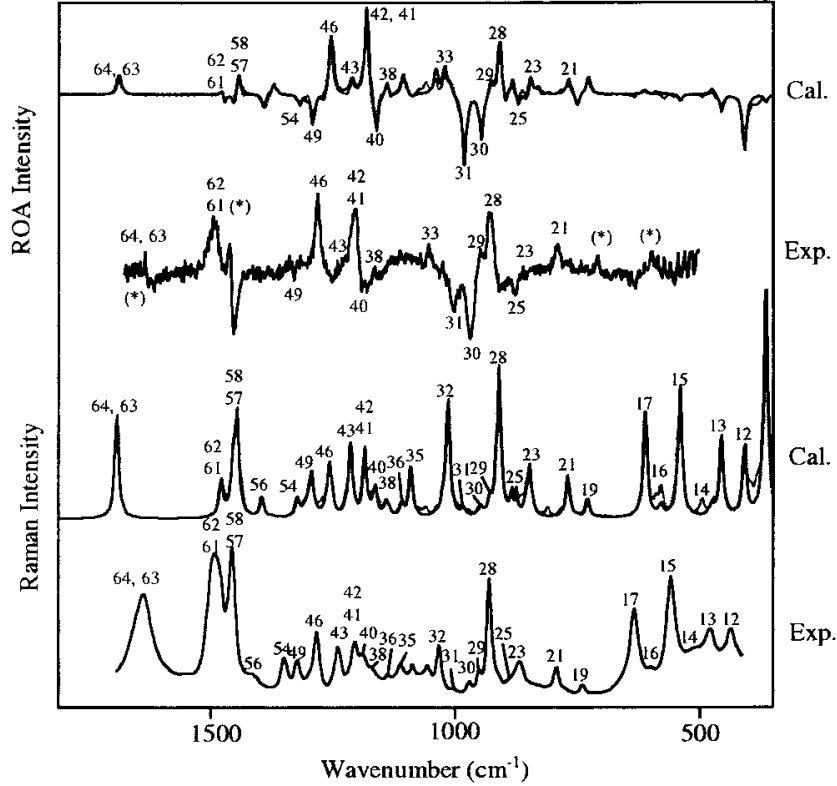

Figure 5. Comparison of simulated Raman and ROA spectra for conformer I (solid line) to the experiment. Regions obscured by artifacts in the experimental ROA spectrum are marked by asterisk $(*)$. The normal mode numbering corresponds to those in Table 5 . The calculated spectra drawn by the dotted line were obtained as a sum of the three conformers (74\% of I, $13 \%$ of II, and $13 \%$ of III).

molecular configuration (L-,L-). ROA intensities of the modes involving the inner six-member ring (amide $\mathbf{I}$, amide $\mathbf{I I}$ in the Table 5) are, as expected, least sensitive to the conformational changes (cf. Figure 4). Most of the modes, however, cannot be localized because of the strong mechanical coupling in the tricyclic system and the exciton coupling between the $C_{2}$ symmetry-related molecular parts.

Since the analysis based on spin-spin coupling constants reliably indicates that about $20 \%$ of proline rings adopt the conformation more deviated from the molecular plane (see Figure 2) and because the relative energies of conformers II and III are approximately equal (Table 2), we consider the conformer populations $74 \%$ of I, $13 \%$ of II, and $13 \%$ of III as the most realistic. This ratio does provide the correct effective population of conformer II obtained by the two state NMR model $(20 \%-13 \%$ of II $+(1 / 2)(13 \%$ of III) $)$. Such a mixture of conformers would not change significantly the ROA spectrum (dotted line in Figure 5), and hence it is consistent also with the ROA data.

\section{Conclusions}

Computed relative energies as well as the NMR and ROA parameters contain incomplete information about molecular conformation, but their combined analysis provided reasonable estimate of the conformer ratios for cyclo(L-Pro-L-Pro) in solution. Both the NMR shielding and coupling constants are sensitive to conformational changes, but for the shielding is this dependence obscured by the influence of the solvent and limited possibilities of the modeling. More reliable conclusions could be drawn from the comparison of the theoretical and experimental spin-spin coupling constants, indicating that about $20 \%$ of the proline residues in the molecule adopt a conformation differing from that found in the crystal. The ab initio modeling of the spin-spin couplings was proved to be a convenient and more universal alternative to the conventional empirical techniques based on the Karplus equations. The results are consistent with the analysis of the Raman and ROA spectra. The vibrational
TABLE 5: Calculated Vibrational Frequencies (Conformer I, in $\mathrm{cm}^{-1}$ ) and Their Assignment to Observed Transitions

\begin{tabular}{|c|c|c|c|c|}
\hline mode & symmetry & $\omega_{\text {calc }^{a}}^{a}$ & $\omega_{\exp }$ & remark \\
\hline 64 & B & 1698.9 & 1635 & amide $\mathbf{I}(\mathrm{C}=\mathrm{O}$ stretch, 2 modes $)$ \\
\hline 63 & A & 1697.2 & & \\
\hline 62 & A & 1483.3 & 1492 & $\mathrm{CH}_{2}$ scissoring (6 modes) \\
\hline 61 & B & 1482.3 & & \\
\hline 60 & B & 1459.3 & & \\
\hline 59 & A & 1459.0 & & \\
\hline 58 & A & 1450.7 & 1457 & \\
\hline 57 & B & 1450.6 & & \\
\hline 56 & A & 1399.9 & 1414 & amide II ( 2 modes) \\
\hline 55 & B & 1378.7 & & \\
\hline 54 & A & 1327.5 & 1350 & $\mathrm{CH}_{2}$ wagging ( 6 modes) \\
\hline 53 & B & 1323.5 & & \\
\hline 52 & B & 1310.2 & & \\
\hline 51 & B & 1301.8 & & \\
\hline 50 & A & 1299.4 & & \\
\hline 49 & A & 1296.2 & 1323 & \\
\hline 48 & B & 1274.6 & & $\mathrm{C}^{\alpha} \mathrm{H}$ bend ( 2 modes) \\
\hline 47 & A & 1263.4 & & \\
\hline 46 & B & 1262.0 & 1284 & complex $\mathrm{CH}$ bend \\
\hline 45 & A & 1257.7 & & \\
\hline 44 & B & 1218.1 & & \\
\hline 43 & A & 1216.1 & 1239 & skeletal deformation \\
\hline 42 & A & 1189.1 & 1205 & amide III + skeletal deformation \\
\hline 41 & B & 1188.3 & & \\
\hline 40 & A & 1167.3 & 1188 & $\mathrm{~N}-\mathrm{C}^{\alpha}$ stretch \\
\hline 39 & B & 1166.7 & & \\
\hline 38 & A & 1144.0 & 1166 & $\mathrm{C}-\mathrm{C}$ and $\mathrm{N}-\mathrm{C}^{\alpha}$ stretch $\mathrm{N}-\mathrm{C}$ \\
\hline 37 & B & 1138.4 & & $\mathrm{~N}-\mathrm{C}^{\alpha}$ stretch \\
\hline 36 & B & 1111.7 & 1138 & $\begin{array}{l}\mathrm{C}-\mathrm{C} \text { stretch, skeletal } \\
\text { deformation ( } 11 \text { modes) }\end{array}$ \\
\hline 35 & A & 1093.5 & 1113 & \\
\hline 34 & B & 1044.0 & & \\
\hline 33 & A & 1025.4 & 1055 & \\
\hline 32 & A & 1016.4 & 1133 & \\
\hline 31 & B & 985.6 & & \\
\hline 30 & B & 949.9 & 970 & \\
\hline 29 & A & 930.3 & & \\
\hline 28 & A & 912.4 & 931 & \\
\hline 27 & B & 902.0 & & \\
\hline 26 & B & 885.8 & & \\
\hline 25 & A & 874.5 & 878 & torsional deformation \\
\hline 24 & B & 857.0 & & $\mathrm{C}-\mathrm{CO}, \mathrm{N}-\mathrm{C}$ stretch \\
\hline 23 & A & 848.8 & 867 & $b$ \\
\hline 22 & B & 832.6 & & $b$ \\
\hline 21 & A & 770.4 & 790 & $b$ \\
\hline 20 & B & 752.4 & & $b$ \\
\hline 19 & A & 729.4 & 739 & $b$ \\
\hline 18 & B & 614.3 & & $b$ \\
\hline 17 & A & 611.4 & 632 & $b$ \\
\hline 16 & B & 578.2 & 598 & $b$ \\
\hline 15 & A & 539.4 & 559 & $b$ \\
\hline 14 & B & 474.2 & 507 & $b$ \\
\hline 13 & A & 454.1 & 482 & $b$ \\
\hline 12 & A & 407.0 & 437 & $b$ \\
\hline 11 & A & 363.2 & & $b$ \\
\hline 10 & B & 327.8 & & $b$ \\
\hline 9 & A & 266.2 & & $b$ \\
\hline 8 & B & 243.8 & & $b$ \\
\hline 7 & A & 215.9 & & $b$ \\
\hline 6 & B & 194.9 & & $b$ \\
\hline 5 & B & 173.1 & & $b$ \\
\hline 4 & A & 108.4 & & $b$ \\
\hline 3 & B & 95.9 & & $b$ \\
\hline 2 & A & 92.9 & & $b$ \\
\hline 1 & A & 45.9 & & $b$ \\
\hline
\end{tabular}

${ }^{a}$ Harmonic force field was calculated at the BPW91/6-311G** level. ${ }^{b}$ Mostly delocalized deformations.

spectra are more limited by experimental noise and computational errors, but they reflect the conformation of entire molecule and are sensitive to its absolute configuration. 
Acknowledgment. The work was supported by grants of the Grant Agency of the Czech Republic (203/01/0031) and Grant Agency of the Academy of Sciences (A4055104), and by the Ministry of Education of the Czech Republic (LN00A032, Center for Complex Molecular Systems and Biomolecules).

\section{References and Notes}

(1) Karplus, M. J. Chem. Phys. 1959, 30, 11. Karplus, M. J. Am. Chem. Soc. 1963, 85, 2870.

(2) Wolinski, K.; Hinton, J. F.; Pulay, P. J. Am. Chem. Soc. 1990, 112,

(3) Ramsey, N. F. Phys. Rev. 1953, 91, 303.

(4) Bouř, P., Buděšínský, M. J. Chem. Phys. 1999, 110, 2836.

(5) Malkin, V. G.; Malkina, O.; Eriksson, L. A.; Salahub, R. D. In Modern Density Functional Theory; Seminario, J. M., Politzer, P., Eds.; Elsevier: Amsterdam, 1995; p 273.

(6) Åstrand, P. O.; Mikkelsen, K. V.; Jørgensen, P.; Ruud, K.; Helgaker, T. J. Chem. Phys. 1998, 108, 2528.

(7) Bose, B.; Zhao, S.; Stenutz, R.; Cloran, F.; Bondo, P. B.; Bondo, G.; Hertz, B.; Carmichael, I.; Serianni, A. S. J. Am. Chem. Soc. 1998, 120 11158 .

(8) Sychrovský, V.; Gräfenstein, J.; Cremer, D. J. Chem. Phys. 2000, 113,3530 . 9402.

(9) Helgaker, T.; Watson, M.; Handy, N. C. J. Chem. Phys. 2000, 113,

(10) Bouř, P. J. Comput. Chem. 2001, 22, 426.

(11) Bell, A. F.; Hecht, L.; Barron, L. D. J. Am. Chem. Soc. 1998, 120, 5820 .

(12) Barron, L. D.; Hecht, L.; Blanch, E. W.; Bell, A. F. Prog. Biophys. Mol. Biol. 2000, 73, 1.

(13) Tam, C. N.; Bouř, P.; Keiderling, T. A. J. Am. Chem. Soc. 1996, 118,10285

(14) Barron, L. D. Molecular Light Scattering and Optical Activity; Cambridge University Press: Cambridge, 1982.

(15) Nafie, L. A.; Che, D. In Modern Nonlinear Optics; Evans, M., Kielich, S., Eds.; John Wiley \& Sons: New York, 1994; Part 3, p 105.
(16) Perdew, J. P.; Wang, Y. Phys. Rev. B 1992, 45, 13244

(17) Frisch M. J.; Trucks, G. W.; Schlegel, H. B.; Scuseria, G. E.; Robb, M. A.; Cheeseman, J. R.; Zakrzewski, V. G.; Montgomery, J. A.; Stratmann, R. E.; Burant J. C.; Dapprich, S.; Millam, J. M.; Daniels, A. D.; Kudin, K. N.; Strain, M. C.; Farkas, O.; Tomasi, J.; Barone, V.; Cossi, M.; Cammi, R.; Mennucci, B.; Pomelli, C.; Adamo, C.; Clifford, S.; Ochterski, J.; Petersson, G. A.; Ayala, P. Y.; Cui, Q.; Morokuma, K.; Malick, D. K.; Rabuck, A. D.; Raghavachari, K.; Foresman, J. B.; Cioslowski, J.; Ortiz, J. V.; Stefanov, B. B.; Liu, G.; Liashenko, A.; Piskorz, P.; Komaroni, I.; Gomperts, R.; Martin, R. L.; Fox, D. J.; Keith, T.; Al-Laham, M. A.; Peng C. Y.; Nanayakkara, A.; Gonzales, C.; Challacombe, M.; Gill, P. M. W.; Johnson, B. G.; Chen, W.; Wong, M. W.; Andres, J. L.; Head-Gordon, M.; Replogle, E. S.; Pople, J. A. Gaussian 98, revisions A.3, A.7, and A.9; Gaussian, Inc.: Pittsburgh, PA, 1998.

(18) Klamt, A.; Schürmann, G. J. Chem. Soc., Perkin Trans. 2 1993, 2, 799.

(19) Becke, A. D. Phys. Rev. A 1998, 38, 3098.

(20) Kraka, E; Grafenstein, J.; Gauss, J.; Reichel, F; Olsson, L; Konkoli, Z.; He, Z.; Cremer, D. Program Package COLOGNE 99; Göteborg University: Göteborg, 1999.

(21) Kutzelnigg, W.; Fleischer, U.; Schindler, M. In NMR-Basic Principles and Progress; Springer: Heidelberg, 1990; Vol. 23, p 165.

(22) Siemion, I. Z. Liebigs. Ann. 1971, 88, 748.

(23) Haasnoot, C. A. G.; de Leeuw, F. A. A. M.; de Leeuw, H. P. M.; Altona, C. Biopolymers; 1981, 20, 1211.

(24) de Leeuw, F. A. A. M.; Altona, C. J. Comput. Chem. 1983, 4 428 .

(25) Haasnoot, C. A. G.; de Leeuw, F. A. A. M.; Altona, C. Tetrahedron 1980, 36, 2783 .

(26) Altona, C.; Geise, H.; Romers, C. Tetrahedron 1968, 24, 13.

(27) Sleeckx, J. J. M.; Anteunis, M. J. O. Bull. Soc. Chim. Belg. 1985, 94, 187.

(28) Bouř, P.; Baumruk, V.; Hanzliková, J. Collect. Czech. Chem. Commun. 1997, 9, 1384

(29) Hanzlíková, J.; Praus, P.; Baumruk, V. J. Mol. Struct. 1999, 481, 431.

(30) Benedetti, E.; Goodman, M.; Marsh, R. E.; Rapoport, H.; Musich, J. A. Cryst. Struct. Commun. 1975, 4, 641 . 